\title{
Analysis of left ventricular mass in untreated men and in men treated with agalsidase- $\beta$ : data from the Fabry Registry
}

\author{
Dominique P. Germain, MD, PhD ${ }^{1}$, Frank Weidemann, MD, PhD², Ademola Abiose, MD², \\ Manesh R. Patel, $\mathrm{MD}^{4}$, Marta Cizmarik, MS5 , J. Alexander Cole, DSc, $\mathrm{MPH}^{5}$, \\ Dana Beitner-Johnson, $\mathrm{PhD}^{5}$, Karelle Benistan, $\mathrm{MD}^{1}$, Gustavo Cabrera, $\mathrm{MD}^{6}$, Joel Charrow, MD', \\ Ilkka Kantola, $\mathrm{MD}^{8}$, Ales Linhart, $\mathrm{MD}^{9}$, Kathy Nicholls, $\mathrm{MD}^{10}$, Markus Niemann, MD², \\ C. Ronald Scott, MD ${ }^{11}$, Katherine Sims, MD ${ }^{12}$, Stephen Waldek, MD ${ }^{13}$, David G. Warnock, MD ${ }^{14}$, \\ and Jörg Strotmann, MD ${ }^{15}$; on behalf of the Fabry Registry
}

\begin{abstract}
Purpose: The aim of this study was to evaluate the progression of left ventricular hypertrophy in untreated men with Fabry disease and to assess the effects of agalsidase- $\beta$ (recombinant human $\alpha$-galactosidase A) on left ventricular hypertrophy.

Methods: Longitudinal Fabry Registry data were analyzed from 115 men treated with agalsidase- $\beta$ ( $1 \mathrm{mg} / \mathrm{kg} / 2$ weeks) and 48 untreated men. Measurements included baseline left-ventricular mass and at least one additional left-ventricular mass assessment over $\geq 2$ years. Patients were grouped into quartiles, based on left-ventricular mass slopes. Multivariate logistic regression analyses identified factors associated with left ventricular hypertrophy progression.
\end{abstract}

Results: For men in whom treatment was initiated at the age of 18 to $<30$ years, mean left ventricular mass slope was $-3.6 \mathrm{~g} /$ year $(n=31)$ compared with $+9.5 \mathrm{~g} /$ year in untreated men of that age $(n=15)$
$(P<0.0001)$. Untreated men had a 3.4-fold higher risk of having faster increases in left-ventricular mass compared with treated men (odds ratio: 3.43; 95\% confidence interval: 1.05-11.22; $P=0.0415$ ). A baseline age of $\geq 40$ years was also associated with left-ventricular hypertrophy progression (odds ratio: 5.03; 95\% confidence interval: $1.03-24.49 ; P=0.0457)$ compared with men younger than 30 years.

Conclusion: Agalsidase- $\beta$ treatment for $\geq 2$ years may improve or stabilize left-ventricular mass in men with Fabry disease. Further investigations may determine whether early intervention and stabilization of LVM are correlated with clinical outcomes.

Genet Med advance online publication 23 May 2013

Key Words: cardiomyopathy; enzyme replacement therapy; Fabry disease; lysosomal storage diseases; recombinant a-galactosidase A
Fabry disease (FD) (OMIM 301500) is a rare disorder in which globotriaosylceramide and other glycosphingolipids accumulate within lysosomes because of insufficient activity of a-galactosidase A. ${ }^{1,2}$ Over time, the glycosphingolipid substrate progressively accumulates within blood vessels and various other cell types throughout the body. The initial signs and symptoms of FD frequently include neuropathic pain in the extremities, hypohidrosis, angiokeratomas, and gastrointestinal discomfort. ${ }^{3}$ Over a period of decades, the accumulation of glycosphingolipids impairs vital organ function, putting patients at risk of developing renal failure, stroke, and cardiovascular dysfunction. ${ }^{4-6}$ Because it is an $\mathrm{X}$-linked disorder, hemizygous males typically experience the most severe manifestations of FD, ${ }^{1}$ but heterozygous females can also develop serious complications. ${ }^{4-7}$

The cardiovascular manifestations of FD can include leftventricular hypertrophy (LVH), atrial and left-ventricular arrhythmias, heart block, valvular dysfunction, angina, and arterial wall thickening. ${ }^{4,5,8-13}$ As the disease progresses, the hypertrophy becomes more severe, including the development of myocardial fibrosis and serious cardiac events including cardiac-related death. $., 5,5,11$

Agalsidase- $\beta$, a recombinant form of human $\alpha$-galactosidase A (Fabrazyme, Genzyme a Sanofi Company, Cambridge, MA), is approved for use as enzyme replacement therapy (ERT) for FD. In clinical studies, agalsidase $-\beta$ at a dose of $1 \mathrm{mg} / \mathrm{kg} / 2$ weeks cleared microvascular endothelial glycosphingolipid deposits from the heart, as well as from kidney and skin, within 5 months. ${ }^{14}$ It also provided long-term stabilization of renal function in patients with mild renal involvement ${ }^{15}$ and delayed time to renal, cardiovascular, and cerebrovascular events in patients with more advanced FD. ${ }^{16}$

Because it is a rare disorder, the randomized clinical trials of agalsidase- $\beta$ included a relatively limited number of subjects with FD. ${ }^{14,16}$ Several prospective, observational studies have reported cardiac data from patients treated with agalsidase- $\beta$, but these also involved only small numbers of patients. ${ }^{17,18}$ 
The Fabry Registry (NCT00196742) is an observational database that compiles clinical data from both treated and untreated patients; it was established to further investigate the long-term effects of ERT and the natural progression of FD in a larger and more diverse patient population. The first objective of the current analyses was to document the progression of LVH in untreated men and in men treated with agalsidase- $\beta$ at a dose of $1 \mathrm{mg} / \mathrm{kg} / 2$ weeks. The second objective was to identify determinants of cardiovascular disease progression in men with FD.

\section{MATERIALS AND METHODS}

Fabry Registry patients provided informed consent through local institutional review boards/ethics committees and could decline to participate or withdraw consent at any time. Because physicians determined which clinical assessments individual patients underwent and the timing of those various assessments, patients' ages and time intervals between assessments were variable.

To be included in the untreated cohort, men must have had a baseline left-ventricular mass (LVM) measurement at the age of 18 years or older and at least one additional LVM measurement over a span of $\geq 2$ years before the start of any agalsidase- $\beta$ treatment. Men in the treated cohort must have received agalsidase- $\beta$ at an average dose at or near the recommended licensed dose of $1 \mathrm{mg} / \mathrm{kg} / 2$ weeks ( $\geq 0.9$ to $\leq 1.1 \mathrm{mg} / \mathrm{kg} / 2$ weeks) and had a baseline LVM measurement at the age of 18 years or older and at least one posttreatment LVM measurement over a span of $\geq 2$ years during the treatment period. If a patient did not have an echocardiogram at the time treatment was initiated, an LVM measurement obtained within the first 6 months of first treatment was used as the baseline value. The observation period extended through 25 June 2009 or until death, if patients died before that date. The cutoff date of 25 June 2009 was used to restrict the analyses to data reported while patients were treated with the recommended licensed dose of agalsidase- $\beta$ $(1 \mathrm{mg} / \mathrm{kg} / 2$ weeks); many patients received temporarily lowered doses after that date, due to an interruption in the manufacturing of agalsidase- $\beta$. Other baseline demographic and clinical data analyzed included age, genotype, body mass index, estimated glomerular filtration rate (eGFR), blood pressure, plasma a-galactosidase activity, urinary protein to creatinine ratio, and reported angiotensin-converting enzyme inhibitor/angiotensin II receptor blocker use (ACEi/ARB). As of the 25 June 2009 cutoff date, 1,426 adult males were enrolled in the Fabry Registry.

Standard two-dimensional or M-mode echocardiographic measurements (parasternal long-axis views) were used to determine left-ventricular end-diastolic dimension (LVed), left-ventricular end-systolic dimension (LVes), left posterior wall thickness (LPWT), septal wall thickness (SWT), left atrial diameter, and aortic root dimension. LVM was calculated as follows: $\mathrm{LVM}=0.8 \times\left(1.04\left([\mathrm{LVed}+\mathrm{LPWT}+\mathrm{SWT}]^{3}-[\mathrm{LVed}]^{3}\right)\right)$ $+0.6 \mathrm{~g} .{ }^{19}$ Because not all men had updated height and weight data recorded in the Fabry Registry at the time of their baseline LVM measurement, LVM was not indexed to these parameters, and $\mathrm{LVH}$ was defined as LVM $\geq 225 \mathrm{~g} .{ }^{20}$ The Fabry Registry recommends that echocardiographic parameters be assessed every 12 months in patients aged 18 years or older. ${ }^{21}$ However, the frequency of echocardiographic assessments varied among patients because treating physicians determined the actual frequency of clinical assessments.

The first two analyses were designed to examine changes in LVM by strata of age and by treatment status. Untreated patients (or patients with echocardiographic data measured before treatment initiation) and patients treated with agalsidase- $\beta$ were separately subgrouped into the following baseline age categories: $18-29,30-39,40-49$, and $\geq 50$ years. Regression slopes representing each patient's change in LVM per year of followup were calculated within each subgroup.

The second analysis was designed to identify additional factors associated with changes in LVM. To achieve this, all patients (treated and untreated) were grouped into quartiles in which quartile $1(\mathrm{Q} 1)$ included patients with the lowest LVM slopes, and quartile $4(\mathrm{Q} 4)$ included patients with the highest LVM slopes. A multivariate logistic regression analysis was used to identify factors associated with men in Q1 compared with men in Q4. Data from patients who reported $\geq 2$ years of LVM data during both the untreated and treated periods were included within each respective observation period, and subjects were nested within each cohort.

Preliminary univariate analyses were conducted to identify individual variables that were associated with change in LVM. Variables in these analyses included baseline age, treatment status, baseline LVM, hypertension, urinary protein to creatinine ratio, baseline creatinine clearance, eGFR, history of arrhythmias, stroke, smoking, elevated cholesterol, ejection fraction, and aortic root diameter for the subgroups of patients who had each type of data available. Results from the multivariate logistic regression model are expressed as odds ratios, with corresponding 95\% confidence intervals (CIs) and $P$ values. An a level of 0.05 was used as the cut point to determine statistical significance. Statistical analyses were performed using SAS statistical software version 9.2 (SAS Institute, Cary, NC).

\section{RESULTS}

Forty-eight men met the inclusion criteria for the untreated cohort, having at least two echocardiographic LVM measurements over a span of $\geq 2$ years $(n=48)$. A total of 115 men met the inclusion criteria for the agalsidase- $\beta$-treated cohort, that includes a baseline LVM and at least one posttreatment LVM measurement over a span of $\geq 2$ years $(n=115)$. Median followup time was 4.1 years in the untreated group (ranging from 2.1 to 12.7 years) and 4.8 years in the agalsidase- $\beta$-treated group (ranging from 1.8 to 9.5 years). Eleven men were included in both cohorts because they had sufficient LVM data during both observation periods. All men included in these analyses had a confirmed diagnosis of FD by either genotype analysis (140 of 152 ) or by measurement of $\alpha$-galactosidase A enzyme activity in plasma or leukocytes (12 of 152).

Baseline demographic and clinical characteristics for men in each cohort are summarized in Table 1. Most parameters were 
Table 1 Baseline demographic and clinical characteristics

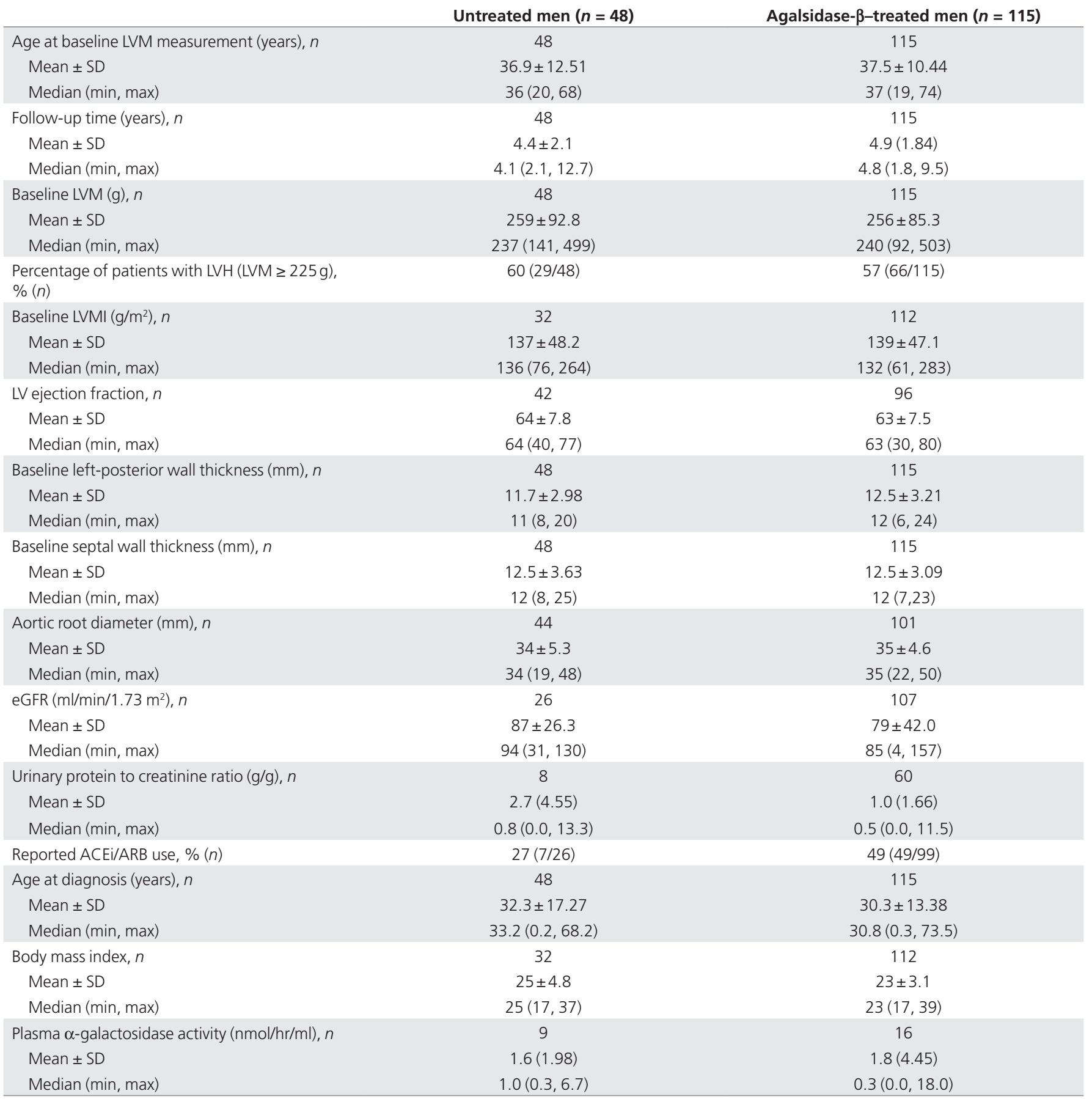

The numbers of patients $(n)$ are different for various clinical parameters because many patients did not have all types of data reported to the Fabry Registry. For untreated patients, baseline represents a patient's earliest assessment data reported to the Fabry Registry. For treated patients, baseline represents the assessment date closest to a patient's first infusion of agalsidase- $\beta$ (within \pm 6 months of the first infusion) for all parameters, except plasma $\alpha$-galactosidase enzyme activity, which was collected at the time of enrollment in the Fabry Registry.

ACEi/ARB, angiotensin-converting enzyme inhibitor or angiotensin receptor blocker; eGFR, estimated glomerular filtration rate, calculated by the Chronic Kidney Disease Epidemiology Collaboration equation; ${ }^{31}$ LVM, left-ventricular mass; LVH, left-ventricular hypertrophy; LVMI, left-ventricular mass index; max, maximum; min, minimum.

similar between the two cohorts, including baseline LVM, age at baseline LVM, age at diagnosis, ejection fraction, LPWT, septal wall thickness, and aortic root diameter. Sixty percent (29 of 48) of men in the untreated cohort had LVH at baseline, as did $57 \%$ (66 of 115) of men in the treated cohort. Mean baseline
LVM index (LVMI) was similar between the two cohorts, although only a subset of patients had such data available. Baseline median urinary protein to creatinine ratio was slightly higher in untreated men; however, data were reported for only 8 of 48 untreated men. Similarly, the status of ACEI or ARB use 
was reported by only 26 of 48 untreated men. The numbers of patients for whom data are reported make it difficult to compare these parameters between treated and untreated cohorts in any meaningful way.

Mean LVM slopes for each baseline age category are shown in Figure 1. LVM progressively increased during the untreated period in men aged $18-29$ years $(n=15), 30-39$ years $(n=17)$, and 40-49 years $(n=7)$ (Figure 1a). By contrast, LVM decreased or remained stable for those who initiated agalsidase- $\beta$ treatment between the ages of 18 and 29 years $(n=31), 30$ and 39 years $(n=44)$, and 40 and 49 years $(n=23)$ (Figure $1 \mathbf{b})$. A summary of regression slopes across age categories is shown in Table 2. For those who initiated treatment between the ages of 18 and 29 years, mean LVM slope was $-3.6 \mathrm{~g} /$ year $(n=31)$ compared with $+9.5 \mathrm{~g} /$ year in untreated men who were in that age category at baseline $(n=15)(P<0.0001)$. LVM slopes also tended to be lower in men who initiated agalsidase- $\beta$ treatment between the ages of 30 and 39 and 40 and 49 compared with untreated men in those age categories; however, these differences did not achieve statistical significance. The mean LVM slope in men who began treatment at the age of $\geq 50$ years was $+7.7 \pm 4.84 \mathrm{~g} /$ year; this was not significantly different than the mean LVM slope in untreated men aged $\geq 50$ years at baseline $(0.4 \pm 9.41 \mathrm{~g} /$ year $)(P=0.484)$.

In the analysis of additional factors associated with changes in LVM, the two groups (48 untreated and 115 agalsidase- $\beta$ treated men) were combined. Q1 included 40 men with the greatest improvement (i.e., declines in LVM), with a mean slope $-1.2 \pm 1.73 \mathrm{~g} /$ year, and Q4 included 41 men with the greatest increases in LVM (mean slope $+9.1 \pm 2.98 \mathrm{~g} /$ year) over the observation period (Table 3). Fifty percent of the men in Q1 had $\mathrm{LVH}$ at baseline (defined as $\mathrm{LVM} \geq 225 \mathrm{~g}$ ), whereas $90 \%$ of men in Q4 had LVH at baseline (Table 3). Men in Q1 who received agalsidase- $\beta$ treatment began treatment at a younger median age than men in Q4 (36 vs. 44 years, respectively). Men in Q1 also initiated treatment sooner after the onset of Fabry symptoms, compared with men in Q4 (median: 19 vs. 31 years).

In preliminary univariate analyses, baseline age category, baseline LVM greater or lower than the median, and treatment status were significantly associated with changes in LVM
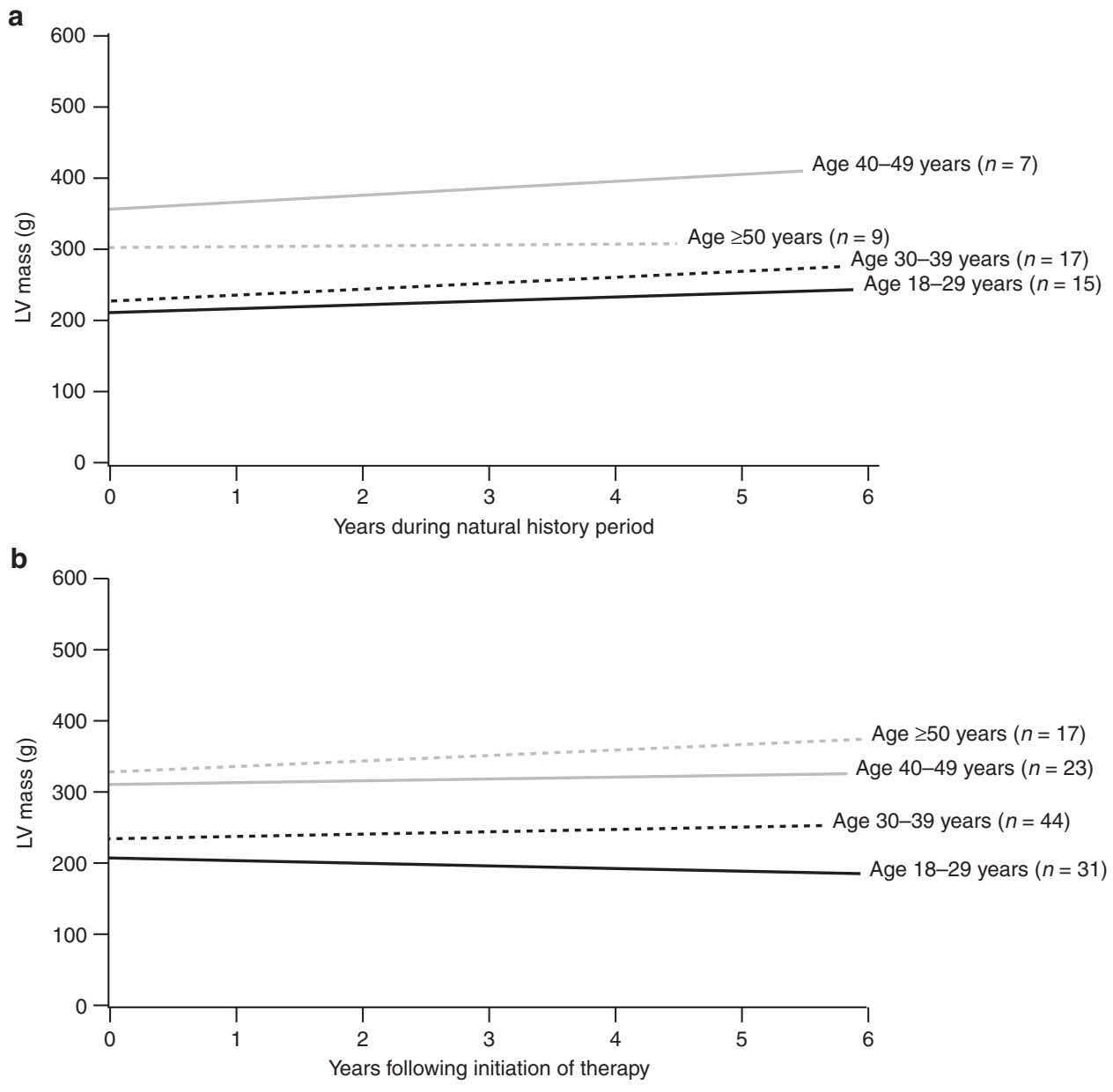

Figure 1 Cardiovascular disease progression in untreated and agalsidase- $\beta$-treated men, by age category at baseline. Patients were grouped into four age categories, as shown. (a) LVM slopes in 48 men during the natural history period; baseline age represents age at first LVM assessment. (b) LVM slopes in 115 men during the agalsidase- $\beta$ treatment period; baseline age represents age at initiation of agalsidase- $\beta$ treatment. Eleven men are included in both panels, because they had at least 2 years of LVM data during each observation period. LV, left-ventricular. 
(data not shown), whereas the other clinical variables listed in the Materials and Methods section were not associated with changes in LVM. Therefore, these factors were used as predictor variables in the final multivariate logistic regression model comparing men in Q1 to men in Q4, as shown in Table 4. In the final analysis of the complete cohort, the following findings were documented. Among the 81 men in Q1 and Q4, those who were older than 40 years at baseline had a fivefold increased risk of the most rapid increase in LVM (i.e., being in Q4) than did men who were younger than 30 years at baseline (odds ratio: 5.03; 95\% CI: 1.03-24.49; $P=0.0457$ ) (Table 4). Men who were untreated had a 3.4-fold increased risk of being in Q4 than men who were treated with agalsidase- $\beta$ (odds ratio: 3.43 ; $95 \% \mathrm{CI}$ :

\begin{tabular}{|c|c|c|}
\hline & $\begin{array}{c}\text { LVM slope } \\
\text { (g/year) } \pm \text { SEM }\end{array}$ & $P$ value \\
\hline \multicolumn{3}{|l|}{ Age $18-29$ years } \\
\hline During untreated period $(n=15)$ & $9.5 \pm 2.36$ & \\
\hline During treatment $(n=31)$ & $-3.6 \pm 1.62$ & \\
\hline Difference in LVM slope & $-13.0 \pm 2.72$ & $<0.0001$ \\
\hline \multicolumn{3}{|l|}{ Age $30-39$ years } \\
\hline During untreated period $(n=17)$ & $8.4 \pm 3.55$ & \\
\hline During treatment $(n=44)$ & $2.8 \pm 2.20$ & \\
\hline Difference in LVM slope & $-5.6 \pm 4.12$ & 0.1760 \\
\hline \multicolumn{3}{|l|}{ Age $40-49$ years } \\
\hline During untreated period $(n=7)$ & $13.4 \pm 6.63$ & \\
\hline During treatment $(n=23)$ & $3.4 \pm 2.87$ & \\
\hline Difference in LVM slope & $-10.0 \pm 7.21$ & 0.1691 \\
\hline \multicolumn{3}{|l|}{ Age $\geq 50$ years } \\
\hline During untreated period $(n=9)$ & $0.4 \pm 9.41$ & \\
\hline During treatment $(n=17)$ & $7.7 \pm 4.48$ & \\
\hline Difference in LVM slope & $7.3 \pm 10.33$ & 0.4843 \\
\hline
\end{tabular}

LVM, left-ventricular mass; SEM, standard error of mean.
1.05-11.22; $P=0.0415)$. Men who had a baseline LVM value greater than the median $(238 \mathrm{~g})$ had a higher risk for more rapid cardiovascular disease progression, although this did not achieve statistical significance in the logistic regression model (odds ratio: 2.95; 95\% CI: 0.93-9.38; $P=0.0665$ ). However, when a stepwise selection was used to determine which risk factors were best fitted for the model, a baseline LVM value greater than the median emerged as a highly significant risk factor for cardiovascular disease progression (odds ratio: 5.04; 95\% CI: 1.87-13.60; $P=0.0014$ ).

Genotype data were available for 140 of the 152 men in this cohort (data not shown), and there were very few patients within any single genotype. Five of the 140 patients had the p.N215S mutation, which has been associated with the cardiac variant of FD. ${ }^{22}$ There were four untreated men with the p.N215S mutation with baseline age and LVM values as follows: 21 years/149g, 24 years/264g, 34 years/198g, 36 years/157g, and one agalsidase- $\beta$-treated patient ( 74 years $/ 256 \mathrm{~g}$ at baseline). The number of such cases was too small to discern any possible trends associated with this mutation and LVM.

\section{DISCUSSION}

One purpose of these analyses was to evaluate the natural progression of cardiovascular disease in untreated men with FD and to assess left-ventricular changes after the initiation of agalsidase- $\beta$ treatment. Our findings demonstrate that LVM progressively increased in untreated men. Men who began receiving agalsidase- $\beta$ before the age of 30 exhibited a statistically significant decrease in LVM compared with age-matched untreated men.

A second purpose was to identify specific factors associated with progression of LVH in these patients. Because baseline age, LVM, and other clinical characteristics were very similar between the 48 untreated men and the 115 men who received agalsidase- $\beta$, all patients were grouped into quartiles based on

Table 3 Cardiac characteristics across LVM slope quartiles (all patients)

\begin{tabular}{lcccc} 
& $\begin{array}{c}\text { Slope quartile } \mathbf{1} \\
(\boldsymbol{n}=\mathbf{4 0})\end{array}$ & $\begin{array}{c}\text { Slope quartile } \mathbf{2} \\
(\boldsymbol{n = 4 1 )}\end{array}$ & $\begin{array}{c}\text { Slope quartile 3 } \\
(\boldsymbol{n}=\mathbf{4 1})\end{array}$ & $\begin{array}{c}\text { Slope quartile } \mathbf{4} \\
(\boldsymbol{n}=\mathbf{4 1})\end{array}$ \\
\hline Number of patients per quartile & & & & 15 \\
$\quad$ During untreated period, $n$ & 7 & 13 & 13 & 26 \\
During agalsidase- $\beta$ treatment, $n$ & 33 & 28 & 28 & 41 \\
LVM slope (random slopes, g/year), $n$ & 40 & 41 & 41 & $9.1(2.98)$ \\
Mean (SD) & $-1.2(1.73)$ & $1.7(0.44)$ & $3.9(0.87)$ & $8.5(5.6,17.5)$ \\
Median (min, max) & $-0.7(-5.5,-0.7)$ & $1.7(1.0,2.6)$ & $3.9(2.7,5.5)$ & 41 \\
Baseline LVM (g) & 40 & 41 & 41 & $299(82.6)$ \\
Mean (SD) & $253(89.4)$ & $220(73.9)$ & $256(87.0)$ & $278(145,499)$ \\
Median (min, max) & $226(92,448)$ & $210(137,451)$ & $235(143,503)$ & $90(37 / 41)$ \\
Percentage of patients with LVH at baseline, \% $(n)$ & $50(20 / 40)$ & $34(14 / 41)$ & $59(24 / 41)$ & $42(17 / 41)$ \\
Percentage with HTN at baseline, \% $(n)$ & $28(11 / 40)$ & $42(17 / 41)$ & $37(15 / 41)$ & $39(13 / 33)$ \\
Reported ACEi/ARB use, \% $(n)$ & $52(16 / 31)$ & $32(9 / 28)$ & $55(18 / 33)$ & \\
\hline
\end{tabular}

Baseline during untreated period $=$ date of first LVM measurement; baseline during agalsidase- $\beta$ treatment $=$ first LVM measurement after $( \pm)$ initiation of treatment. HTN $=$ $(\mathrm{SBP} \geq 130$ or $\mathrm{DBP} \geq 80)$; $\mathrm{LVH}=(\mathrm{LVM} \geq 225 \mathrm{~g})$.

ACEi/ARB, angiotensin-converting enzyme inhibitor or angiotensin receptor blocker; DBP, diastolic blood pressure; HTN, hypertension; LVH, left-ventricular hypertrophy; LVM, left-ventricular mass; SBP, systolic blood pressure. 


\begin{tabular}{|c|c|c|}
\hline & $\begin{array}{l}\text { Odds ratio } \\
(95 \% \mathrm{Cl})\end{array}$ & $P$ value \\
\hline $\begin{array}{l}\text { Baseline age ( }<30 \text { years } \\
\text { vs. } \geq 40 \text { years) }\end{array}$ & $5.03(1.03,24.49)$ & 0.0457 \\
\hline $\begin{array}{l}\text { Baseline age ( } 30 \text { to }<40 \text { years } \\
\text { vs. } \geq 40 \text { years) }\end{array}$ & $2.59(0.85,7.89)$ & 0.0935 \\
\hline Received agalsidase- $\beta$ treatment & $3.43(1.05,11.22)$ & 0.0415 \\
\hline $\begin{array}{l}\text { LVM > median at baseline } \\
(>238 \mathrm{~g})^{*}\end{array}$ & $2.95(0.93,9.38)$ & 0.0665 \\
\hline
\end{tabular}

Analyses were based on $n=40$ men in LVM slope Q1 and $n=41$ men in LVM slope Q4.

$\mathrm{Cl}$, confidence interval; LVM, left ventricular mass; Q, quartile.

${ }^{*}$ A stepwise procedure revealed LVM $>$ median to be significant $(P=0.0014)$.

individual LVM slopes. Logistic regression modeling of LVM slope quartiles demonstrated that the following factors were significantly associated with decreases in LVM: younger age at baseline, receiving agalsidase- $\beta$ treatment, and in the stepwise analysis, LVM $<$ median at baseline.

These findings are consistent with results from a prospective, observational study of 32 patients who were treated with agalsidase- $\beta$ for 3 years at a dose of $1 \mathrm{mg} / \mathrm{kg} / 2$ weeks. ${ }^{18}$ Long-term agalsidase- $\beta$ treatment ( 3 years) was associated with an improvement in regional myocardial function as measured by radial and longitudinal systolic strain. ${ }^{18}$ In a subgroup of 12 patients who did not exhibit myocardial fibrosis at baseline, mean LVM was significantly decreased after 3 years of treatment (baseline age: $36 \pm 4$ years $($ mean $\pm \mathrm{SD})) .{ }^{18}$ However, patients who had already developed myocardial fibrosis before treatment initiation showed little or no improvement in LVH or myocardial function. ${ }^{18}$ Similarly, a significant decrease in mean LVM was reported in 11 patients who were treated with agalsidase- $\beta$ at $1 \mathrm{mg} / \mathrm{kg} / 2$ weeks in an open-label study for a mean duration of 3.8 years (eight men and three women; age 23-54 years at baseline). ${ }^{23}$ An earlier open-label study reported a significant decrease in LVMI in nine patients who received agalsidase- $\beta$ at $1 \mathrm{mg} / \mathrm{kg} / 2$ weeks for 1 year. ${ }^{24}$ The current findings are also generally similar to those of a previous study of LVMI slope in 39 untreated men and 39 untreated women with FD. ${ }^{25}$ Among the untreated men, LVMI increased over time in all age categories, with the greatest increases reported in men over the age of 40 years at baseline. ${ }^{25}$ It should be noted that the studies of Weidemann et al. ${ }^{18}$ and Imbriaco et al. ${ }^{23}$ used cardiac magnetic resonance imaging (MRI), which provides higher spatial resolution than the standard echocardiographic data reported here.

The LVM slopes observed in the oldest group of men are somewhat difficult to interpret; men who initiated treatment over the age of 50 years exhibited a mean increase in LVM over time, whereas untreated men who were over the age of 50 years at the time of their first LVM measurement showed a mean decrease in LVM over time. However, there was not a statistically significant difference between the mean slopes of treated versus untreated men in this age group. It is possible that the increase in LVM observed in men who initiated agalsidase- $\beta$ treatment after the age of 50 years may be associated with a relatively advanced stage of disease, where cardiac hypertrophy cannot be prevented or reversed by ERT. This would be consistent with the growing body of evidence that ERT may be most beneficial in patients who have not yet developed substantial myocardial fibrosis. ${ }^{18,26}$ The observed decrease in LVM among the older untreated men could reflect an even more advanced stage of myocardial fibrosis. In the late stages of transmural fibrosis associated with hypertrophy, cardiomyocyte death can induce scar tissue and thinning of the left-ventricular posterolateral wall. ${ }^{27}$ However, the issue of fibrosis cannot be addressed from the available echocardiographic data. Higher resolution imaging assessments, such as MRI or autopsy data, would be needed to determine the underlying cardiac histopathology in these patients.

Agalsidase alfa (recommended licensed dose: $0.2 \mathrm{mg} / \mathrm{kg} / 2$ weeks) is another form of recombinant human a-galactosidase A (Shire Human Genetic Therapies, Cambridge, MA). Mehta et al. ${ }^{28}$ reported a modest reduction in LVMI in patients from the Fabry Outcome Survey observational database who had LVH and were treated with agalsidase alfa at a dose of $0.2 \mathrm{mg} / \mathrm{kg} / 2$ weeks for 5 years. However, LVMI slopes were not calculated; data were analyzed as change from baseline after each year of treatment, with the number of patients $(n)$ ranging from 23 to 32 after each year. ${ }^{28}$ It is difficult to compare those findings with the current LVM slope data, because Mehta et al. ${ }^{28}$ pooled echocardiographic data from men and women and grouped patients by baseline LVH status.

Various limitations are associated with analyzing observational data from a rare-disease registry. Important limitations include the bias related to treatment decisions and the potential for unmeasured confounders to affect measured outcomes, although the multivariable model accounted for the known effectors. Another limitation is the problem of missing data. Although the Fabry Registry provides a schedule of recommended clinical assessments, ${ }^{21}$ treating physicians determine the actual frequency of assessments according to individual patients' needs. Of the 1,426 adult men enrolled in the Fabry Registry as of 25 June 2009, only 152 had reported sufficient longitudinal echocardiographic data to be included in these analyses; this may not be a representative sample of the overall population of patients with FD. Various other types of clinical data, including urinary protein data, ACEI or ARB use, and genotype data were missing for many of the 152 men with longitudinal echocardiographic data. In addition, LVM measured by echocardiography is a less reliable way to assess LVH as compared with LVMI because it is not indexed to body size. Another factor to be considered is that most adult men in the Fabry Registry who had regular echocardiographic assessments did not remain untreated for $\geq 2$ years; many enrolled in the Fabry Registry at the time they initiated agalsidase- $\beta$ treatment. Therefore, the untreated population was substantially smaller than the agalsidase- $\beta$-treated population in these analyses. Finally, it has not been directly demonstrated that a reduction in LVM is associated with better cardiac or general clinical outcomes in Fabry patients. However, LVH is a significant risk 
factor for the occurrence of clinical events in untreated patients with FD, ${ }^{13}$ and elevated LVM is associated with adverse clinical outcomes in patients with chronic kidney disease stages $3-5 .^{29}$ Despite the various limitations, to date, this analysis of registry data represents the largest sample of men with FD treated with agalsidase- $\beta$ who had $\geq 2$ years of longitudinal follow-up reported.

Taken together, these findings suggest that agalsidase- $\beta$ treatment is associated with improvements in LVH when treatment is initiated during the early stages of Fabry cardiomyopathy. Of note, agalsidase- $\beta$ has been similarly shown to be most beneficial, in terms of renal function, when initiated before the onset of advanced renal disease..$^{15,16,30}$ It is important to note that agalsidase- $\beta$ was not an approved therapy at the time that most of these patients began experiencing disease symptoms. Therefore, most men in this cohort did not begin receiving agalsidase- $\beta$ until many years after the onset of their Fabry symptoms (median: 19 years in Q1 and 31 years in Q4). With increased awareness of FD and the availability of ERT, younger patients who begin agalsidase- $\beta$ treatment earlier during the course of disease progression might be expected to experience a more favorable effect on left-ventricular geometry. Future research efforts should be aimed at delineating the relationship between early treatment and long-term clinical cardiovascular outcomes.

\section{ACKNOWLEDGMENTS}

The authors thank the many patients who have agreed to participate in the Fabry Registry as well as the physicians and research coordinators who have entered clinical data of these patients. The authors also thank Albert A. Hagege (Hôpital Européen Georges Pompidou, Paris, France) for expert analysis of echocardiographic data, Judy Kempf (Genzyme, Cambridge, MA) for critical review of the data analyses and helpful discussions, Christoph Wanner (University of Würzburg, Würzburg, Germany) for critical review of the manuscript, and Badari Gudivada (Genzyme) for statistical programming support.

\section{DISCLOSURE}

Genzyme Corporation sponsors the Fabry Registry. Members of the Fabry Registry Board of Advisors include D.P.G., F.W., A.A., M.R.P., G.C., J.C., A.L., M.R.P., C.R.S., K.S., S.W., and D.G.W. Authors who have received research funds or travel support from Genzyme include D.P.G., M.R.P., S.W., J.S., and D.G.W. Authors who have received speaking fees from Genzyme include D.P.G., S.W., and J.S. M.C., A.C., and D.B.J. are Genzyme employees. D.G.W. has served as a paid consultant to Genzyme and also has consultancies and has received travel funds from Abbot, Amgen, Amicus, Gilead, Parion, Relypsa, and Shire. The results presented in this article have not been published previously in whole or part, except in abstract format.

\section{REFERENCES}

1. Desnick RJ, loannou YA, Eng CM. Alpha-galactosidase a deficiency: Fabry disease. In: Scriver C, Beaudet A, Sly W, Valle D (eds). The Metabolic Bases of Inherited Disease, 8th edn. McGraw-Hill: New York, 2001:3733-3774.

2. Germain DP. Fabry disease. Orphanet J Rare Dis 2010;5:30.
3. Hopkin RJ, Bissler J, Banikazemi M, et al. Characterization of Fabry disease in 352 pediatric patients in the Fabry Registry. Pediatr Res 2008;64:550-555.

4. Schiffmann R, Warnock DG, Banikazemi M, et al. Fabry disease: progression of nephropathy, and prevalence of cardiac and cerebrovascular events before enzyme replacement therapy. Nephrol Dial Transplant 2009;24:2102-2111.

5. Wilcox WR, Oliveira JP, Hopkin RJ, et al. Females with Fabry disease frequently have major organ involvement: lessons from the Fabry Registry. Mol Genet Metab 2008;93:112-128.

6. Sims K, Politei J, Banikazemi M, Lee P. Stroke in Fabry disease frequently occurs before diagnosis and in the absence of other clinical events: natural history data from the Fabry Registry. Stroke 2009;40:788-794.

7. Ortiz A, Cianciaruso B, Cizmarik M, et al. End-stage renal disease in patients with Fabry disease: natural history data from the Fabry Registry. Nephrol Dial Transplant 2010;25:769-775.

8. Senechal M, Germain DP. Fabry disease: a functional and anatomical study of cardiac manifestations in 20 hemizygous male patients. Clin Genet 2003; 63:46-52.

9. Boutouyrie P, Laurent S, Laloux B, Lidove O, Grunfeld JP, Germain DP. Arterial remodelling in Fabry disease. Acta Paediatr Supp/ 2002;91:62-66.

10. Pierre-Louis B, Kumar A, Frishman WH. Fabry disease: cardiac manifestations and therapeutic options. Cardiol Rev 2009;17:31-35.

11. Shah JS, Hughes DA, Sachdev B, et al. Prevalence and clinical significance of cardiac arrhythmia in Anderson-Fabry disease. Am J Cardio/ 2005;96:842-846.

12. Weidemann F, Strotmann JM, Niemann $M$, et al. Heart valve involvement in Fabry cardiomyopathy. Ultrasound Med Bio/ 2009;35:730-735.

13. Patel MR, Cecchi F, Cizmarik M, et al. Cardiovascular events in patients with fabry disease natural history data from the Fabry registry. J Am Coll Cardiol 2011;57:1093-1099.

14. Eng CM, Guffon N, Wilcox WR, et al.; International Collaborative Fabry Disease Study Group. Safety and efficacy of recombinant human alpha-galactosidase A-replacement therapy in Fabry's disease. N Engl J Med 2001;345:9-16.

15. Germain DP, Waldek S, Banikazemi M, et al. Sustained, long-term renal stabilization after 54 months of agalsidase beta therapy in patients with Fabry disease. J Am Soc Nephro/ 2007;18:1547-1557.

16. Banikazemi M, Bultas J, Waldek S, et al.; Fabry Disease Clinical Trial Study Group. Agalsidase-beta therapy for advanced Fabry disease: a randomized trial. Ann Intern Med 2007;146:77-86.

17. Weidemann F, Breunig F, Beer M, et al. Improvement of cardiac function during enzyme replacement therapy in patients with Fabry disease: a prospective strain rate imaging study. Circulation 2003;108:1299-1301.

18. Weidemann F, Niemann M, Breunig F, et al. Long-term effects of enzyme replacement therapy on Fabry cardiomyopathy: evidence for a better outcome with early treatment. Circulation 2009;119:524-529.

19. Lang RM, Bierig M, Devereux RB, et al.; Chamber Quantification Writing Group; American Society of Echocardiography's Guidelines and Standards Committee; European Association of Echocardiography. Recommendations for chamber quantification: a report from the American Society of Echocardiography's Guidelines and Standards Committee and the Chamber Quantification Writing Group, developed in conjunction with the European Association of Echocardiography, a branch of the European Society of Cardiology. J Am Soc Echocardiogr 2005;18:1440-1463.

20. Lang RM, Bierig M, Devereux RB, et al.; American Society of Echocardiography's Nomenclature and Standards Committee; Task Force on Chamber Quantification; American College of Cardiology Echocardiography Committee; American Heart Association; European Association of Echocardiography, European Society of Cardiology. Recommendations for chamber quantification. Eur J Echocardiogr 2006;7:79-108.

21. Fabry Registry Recommended Schedule of Assessments. https://www. Isdregistry.net/fabryregistry/. Accessed 11 March 2013.

22. Meehan SM, Junsanto T, Rydel JJ, Desnick RJ. Fabry disease: renal involvement limited to podocyte pathology and proteinuria in a septuagenarian cardiac variant. Pathologic and therapeutic implications. Am J Kidney Dis 2004;43:164-171.

23. Imbriaco M, Pisani A, Spinelli L, et al. Effects of enzyme-replacement therapy in patients with Anderson-Fabry disease: a prospective long-term cardiac magnetic resonance imaging study. Heart 2009;95:1103-1107.

24. Spinelli L, Pisani A, Sabbatini M, et al. Enzyme replacement therapy with agalsidase beta improves cardiac involvement in Fabry's disease. Clin Genet 2004;66:158-165.

25. Kampmann $C$, Linhart A, Baehner $F$, et al. Onset and progression of the Anderson-Fabry disease related cardiomyopathy. Int J Cardiol 2008;130: 367-373. 
26. Beer M, Weidemann F, Breunig F, et al. Impact of enzyme replacement therapy on cardiac morphology and function and late enhancement in Fabry's cardiomyopathy. Am J Cardiol 2006;97:1515-1518.

27. Hasegawa H, Takano H, Shindo S, et al. Images in cardiovascular medicine. Transition from left ventricular hypertrophy to massive fibrosis in the cardiac variant of Fabry disease. Circulation 2006;113:e720-e721.

28. Mehta A, Beck M, Elliott P, et al.; Fabry Outcome Survey investigators. Enzyme replacement therapy with agalsidase alfa in patients with Fabry's disease: an analysis of registry data. Lancet 2009;374:1986-1996.

29. Chen SC, Chang JM, Liu WC, et al. The ratio of observed to predicted left ventricular mass is independently associated with increased cardiovascular events in patients with chronic kidney disease. Hypertens Res 2012;35:832-838.
30. Warnock DG, Ortiz A, Mauer M, et al.; Fabry Registry. Renal outcomes of agalsidase beta treatment for Fabry disease: role of proteinuria and timing of treatment initiation. Nephrol Dial Transplant 2012;27:1042-1049.

31. Levey AS, Stevens LA, Schmid CH, et al.; CKD-EPI (Chronic Kidney Disease Epidemiology Collaboration). A new equation to estimate glomerular filtration rate. Ann Intern Med 2009;150:604-612.

(c) (i) This work is licensed under a Creative License. To view a copy of this license, visit http:// creativecommons.org/licenses/by/3.0/deed.en_US 